PROCEEDINGS OF THE

AMERICAN MATHEMATICAL SOCIETY

Volume 129, Number 11, Pages 3241-3252

S 0002-9939(01)06010-5

Article electronically published on April 2, 2001

\title{
AREAS OF TWO-DIMENSIONAL MODULI SPACES
}

\author{
TOSHIHIRO NAKANISHI AND MARJATTA NÄÄTÄNEN
}

(Communicated by Albert Baernstein II)

\begin{abstract}
Wolpert's formula expresses the Weil-Petersson 2-form in terms of the Fenchel-Nielsen coordinates in case of a closed or punctured surface. The area-form in Fenchel-Nielsen coordinates is invariant under the mapping class group on each 2-dimensional Teichmüller space of a surface with singularities, hence areas with respect to it can be calculated for 2-dimensional moduli spaces in cases when the Teichmüller space admits global Fenchel-Nielsen coordinates: The area of the moduli space for the signature $\left(0 ; 2 \theta_{1}, 2 \theta_{2}, 2 \theta_{3}, 2 \theta_{4}\right)$ is $2\left(\pi^{2}-\theta_{1}^{2}-\theta_{2}^{2}-\theta_{3}^{2}-\theta_{4}^{2}\right)$, the definition of signature is generalized to include punctures, cone points and geodesic boundary curves. In case the surface is represented by a Fuchsian group, the area is the classical Weil-Petersson area.
\end{abstract}

\section{INTRODUCTION}

By a hyperbolic surface $R$ we mean a 2-dimensional cone manifold, that is, a surface equipped with a metric of constant curvature -1 which admits conical singular points (or simply cone-points) and all of whose boundary components are totally geodesic closed curves. In polar co-ordinates around a cone-point, the metric has the form $d r^{2}+\sinh ^{2} r d \varphi^{2}$ where $r$ is the distance from the cone-point, $\varphi$ is the angular measure around the cone-point, which is measured modulo $\theta$ for some $\theta \in(0,2 \pi)$. This $\theta$ is called the angle around the cone-point. We assume that $R$ has a finite area. Then $R$ has a finite number of singularities, which are, in our notation, cone-points, punctures and boundary curves. We assign the number $\theta$ to a cone-point if the angle around it is $\theta$, the number 0 to a puncture and the number $i \theta$ to a boundary curve if its length is $\theta$. If $R$ has genus $g$ and the numbers $\theta_{1}, \ldots, \theta_{n}$ assigned to its singularities in the way specified above, we call the tuple $\left(g ; \theta_{1}, \ldots, \theta_{n}\right)$ the signature of $R$. Although this definition of signature is not the usual one, it is convenient since it allows us to treat hyperbolic surfaces in a unified manner.

If the Teichmüller space of a hyperbolic orbifold $R$ is two-dimensional, the signature is either of type $(1 ; \theta)$ or of type $\left(0 ; \theta_{1}, \theta_{2}, \theta_{3}, \theta_{4}\right)$ [14, 34.3]. (Remember that the boundary curves have specified lengths.) If the Teichmüller space is parameterized by a Fenchel-Nielsen coordinate system $(l, s), l>0,-\infty<s<\infty$, then the 2 -form

$$
\omega_{W P}=d l \wedge d s
$$

Received by the editors September 23, 1999 and, in revised form, March 9, 2000. 1991 Mathematics Subject Classification. Primary 32G15, 30F35, 57M50. 
is invariant under the mapping class group, and hence it can be considered in the moduli space. The form (1.1) is Wolpert's formula for the Weil-Petersson 2-form 12, Theorem 1.3].

In this paper, we compute the areas of 2-dimensional moduli spaces of hyperbolic cone-surfaces with respect to the form (1.1) when corresponding Teichmüller spaces admit global Fenchel-Nielsen coordinates. The invariance of (1.1) under the mapping class group is proved in Section 3. For the signature $(1 ; 0)$, Wolpert [10] obtained the (Weil-Petersson) area $\pi^{2} / 6$. In 8 we found that the area for the signature $(1 ; 2 \theta), \theta \in[0, \pi) \cup i \mathbf{R}_{+}$is $\frac{1}{6}\left(\pi^{2}-\theta^{2}\right)$. The result is stated only for $\theta \in\{\pi / p: p=2,3, \ldots, \infty\} \cup i \mathbf{R}_{+}$, but the argument applies for other values of $\theta \in[0, \pi) \cup i \mathbf{R}_{+}$as well. See also Section 5. In order to calculate the area, we used the classical result in 3 that the Teichmüller space for the signature $(1 ; 2 \theta)$ is represented by the sublocus of the equation

$$
x^{2}+y^{2}+z^{2}-2 x y z-\sin ^{2} \frac{\theta}{2}=0
$$

satisfying $x, y, z>1$. In this paper we calculate the area of the moduli space for the signature $\left(0 ; 2 \theta_{1}, 2 \theta_{2}, 2 \theta_{3}, 2 \theta_{4}\right)$. We derive in Lemma 2.1 an algebraic equation which represents the Teichmüller space $\mathbf{T}\left(0 ; 2 \theta_{1}, 2 \theta_{2}, 2 \theta_{3}, 2 \theta_{4}\right)$ for the same signature. After considering the action of the mapping class group in Section 3, in Section 4 we prove:

Theorem 1.1. If $\theta_{1}, \theta_{2}, \theta_{3}$ and $\theta_{4}$ are numbers in $[0, \pi / 2] \cup i \mathbf{R}_{+}$, then the area of the moduli space for the signature $\left(0 ; 2 \theta_{1}, 2 \theta_{2}, 2 \theta_{3}, 2 \theta_{4}\right)$ is

$$
2\left(\pi^{2}-\theta_{1}^{2}-\theta_{2}^{2}-\theta_{3}^{2}-\theta_{4}^{2}\right) .
$$

Here the moduli space means $\mathbf{T}\left(0 ; 2 \theta_{1}, 2 \theta_{2}, 2 \theta_{3}, 2 \theta_{4}\right) / \mathcal{M C}$, where $\mathcal{M} C$ denotes the mapping class group of isotopy classes of homeomorphisms (including orientation-reversing ones) fixing each singularity pointwise. Thus, if two of $\theta_{1}, \theta_{2}, \theta_{3}$ and $\theta_{4}$ are identical, $\mathcal{M C}$ is not the full mapping class group. We remark also that $(0 ; \pi, \pi, \pi, \pi)$ is treated here as a degenerate case: the surface of this signature is realized as a parabolic orbifold, rather than a hyperbolic orbifold [4, p. 142]. This case has Weil-Petersson area 0, which will be used in Section 5 to evaluate a constant.

In this paper we treat the case where $\theta_{1}, \theta_{2}, \theta_{3}$ and $\theta_{4}$ are numbers in $[0, \pi / 2]$. Other cases where some $\theta_{i} \in i \mathbf{R}_{+}$follow the same way by slight modifications of matrices and figures in Section 2. Since we make use of traces of matrices in $S L_{2}(\mathbf{R})$ (see Lemma 2.1), noticing that $\cos i \theta=\cosh \theta$, we need no changes in the computation achieved in Section 4.

In [5, 9] and [13], Weil-Petersson volumes of higher-dimensional moduli spaces of punctured surfaces are obtained.

\section{A parameter space for Teichmüller space}

Let $L_{0}$ and $L_{1}$ denote (resp.) the hyperbolic lines formed by the circle $|z|=1$ and the circle $|z|=e^{l / 2}, l \geq 0$. Choose a point $P=-u+i v \in L_{0}, 0 \leq u \leq 1, v=$ $\sqrt{1-u^{2}}$. Let $L_{2}$ denote a hyperbolic line which meets $L_{0}$ at $P$ and intersects $L_{1}$, also. We assume that $L_{2}$ does not meet $L_{1}$ in the right half plane: If the angle from $L_{0}$ to $L_{2}$ is $\theta_{1}$ and the angle from $L_{2}$ to $L_{1}$ is $\theta_{2}$ (see Figure 1a), this assumption 


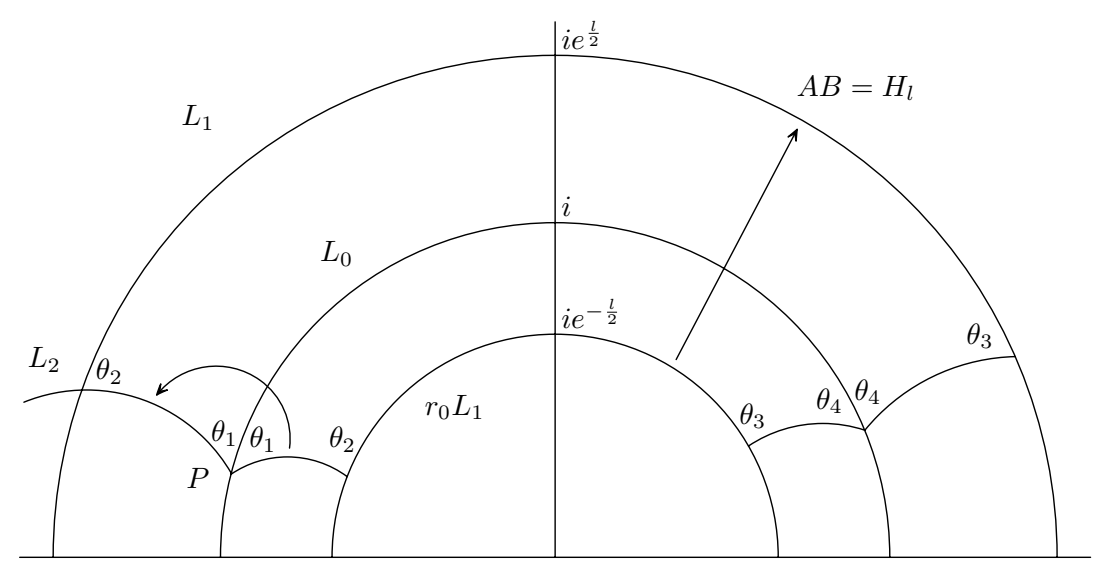

Figure 1a.

is equivalent to

$$
\cos \theta_{1}+\cosh \frac{l}{2} \cos \theta_{2} \geq 0 .
$$

Let $A=r_{2} r_{0}$ and $H_{l}=r_{1} r_{0}$, where $r_{0}, r_{1}$ and $r_{2}$ denote (resp.) reflections in $L_{0}, L_{1}$ and $L_{2}$. They have the matrix-representations

$$
\begin{aligned}
A & =\left(\begin{array}{cc}
\cos \theta_{1}-u v^{-1} \sin \theta_{1} & v^{-1} \sin \theta_{1} \\
-v^{-1} \sin \theta_{1} & \cos \theta_{1}+u v^{-1} \sin \theta_{1}
\end{array}\right), \\
H_{l} & =\left(\begin{array}{cc}
-e^{l / 2} & 0 \\
0 & -e^{-l / 2}
\end{array}\right) .
\end{aligned}
$$

The matrix for $B=A^{-1} H_{l}$ is

$$
B=\left(\begin{array}{cc}
-e^{l / 2}\left(\cos \theta_{1}+u v^{-1} \sin \theta_{1}\right) & e^{-l / 2} v^{-1} \sin \theta_{1} \\
-e^{l / 2} v^{-1} \sin \theta_{1} & -e^{-l / 2}\left(\cos \theta_{1}-u v^{-1} \sin \theta_{1}\right)
\end{array}\right) .
$$

Since the (1,2)-entry is non-negative and $B=r_{0} r_{2} r_{0} \cdot r_{0} r_{1} r_{0}$ is the positive rotation of angle $2 \theta_{2}$ about the intersection point of $r_{0} L_{1}$ and $r_{0} L_{2}$, we know $\operatorname{tr} B \geq 0$. Thus $\operatorname{tr} B=2 \cos \theta_{2}$ and we get

$$
\begin{aligned}
& u=\frac{\left|\cos \theta_{2}+\cosh \frac{l}{2} \cos \theta_{1}\right|}{\left(\cosh ^{2} \frac{l}{2}+2 \cosh \frac{l}{2} \cos \theta_{1} \cos \theta_{2}+\cos ^{2} \theta_{1}+\cos ^{2} \theta_{2}-1\right)^{1 / 2}}, \\
& v=\frac{\sinh \frac{l}{2} \sin \theta_{1}}{\left(\cosh ^{2} \frac{l}{2}+2 \cosh \frac{l}{2} \cos \theta_{1} \cos \theta_{2}+\cos ^{2} \theta_{1}+\cos ^{2} \theta_{2}-1\right)^{1 / 2}} .
\end{aligned}
$$

The Fenchel-Nielsen deformation. Let $\theta_{1}, \theta_{2}, \theta_{3}$ and $\theta_{4}$ be numbers in $[0, \pi / 2]$. Then any two of them satisfy the inequality (2.1) for all $l>0$. Thus the numerator of the first expression in (2.4) does not need absolute-values. We define $A$ and $B$ by $(2.2),(2.3)$ and $(2.4)$ and $\tilde{A}, \tilde{B}$ similarly but replacing $\left(\theta_{1}, \theta_{2}\right)$ by $\left(\theta_{4}, \theta_{3}\right)$. We take conjugations of $\tilde{A}^{-1}$ and $\tilde{B}^{-1}$ by $H_{s} r_{0} E$, where $E(z)=-1 / z, H_{s}(z)=e^{s} z$, to 


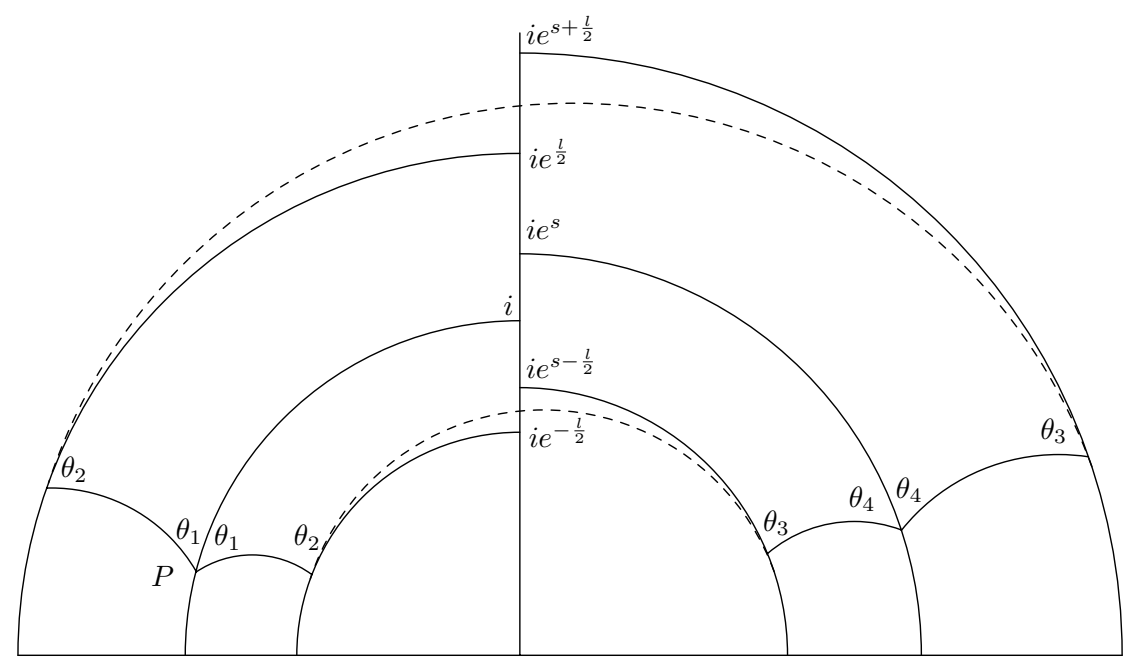

FiguRE 1b.

obtain the transformations $D$ and $C$ with matrix-representations

$$
\begin{aligned}
C & =\left(\begin{array}{cc}
-e^{-l / 2}\left(\cos \theta_{4}-\xi \eta^{-1} \sin \theta_{4}\right) & e^{s} e^{-l / 2} \eta^{-1} \sin \theta_{4} \\
-e^{-s} e^{l / 2} \eta^{-1} \sin \theta_{4} & -e^{l / 2}\left(\cos \theta_{4}+\xi \eta^{-1} \sin \theta_{4}\right)
\end{array}\right), \\
D & =\left(\begin{array}{cc}
\cos \theta_{4}+\xi \eta^{-1} \sin \theta_{4} & e^{s} \eta^{-1} \sin \theta_{4} \\
-e^{-s} \eta^{-1} \sin \theta_{4} & \cos \theta_{4}-\xi \eta^{-1} \sin \theta_{4}
\end{array}\right)
\end{aligned}
$$

where

$$
\begin{aligned}
& \xi=\frac{\cos \theta_{3}+\cosh \frac{l}{2} \cos \theta_{4}}{\left(\cosh ^{2} \frac{l}{2}+2 \cosh \frac{l}{2} \cos \theta_{3} \cos \theta_{4}+\cos ^{2} \theta_{3}+\cos ^{2} \theta_{4}-1\right)^{1 / 2}}, \\
& \eta=\frac{\sinh \frac{l}{2} \sin \theta_{4}}{\left(\cosh ^{2} \frac{l}{2}+2 \cosh \frac{l}{2} \cos \theta_{3} \cos \theta_{4}+\cos ^{2} \theta_{3}+\cos ^{2} \theta_{4}-1\right)^{1 / 2}} .
\end{aligned}
$$

The performed Fenchel-Nielsen deformation is represented in Figure 1a with coordinates $(l, 0)$ and in Figure $1 \mathrm{~b}$ with coordinates $(l, s)$. Since $A B=H_{l}$ and $C D=H_{l}^{-1}, A B C D=1$. We think of $A, B, C$ and $D$ as matrix-valued functions defined in the Fenchel-Nielsen coordinate-space $(l, s) \in \mathbf{R}_{+} \times \mathbf{R}$ for the Teichmüller space of signature $\left(0 ; 2 \theta_{1}, 2 \theta_{2}, 2 \theta_{3}, 2 \theta_{4}\right)$. The transformations $A, B$ and $C$ are (resp.) positive rotations of angles $2 \theta_{1}, 2 \theta_{2}$ and $2 \theta_{3}$ about their fixed points. Then, since $\operatorname{tr} A B=-2 \cosh \frac{l}{2} \leq-2$, symmetry (by replacing $(A, B, C, D)$ with $\left(B, C, A, A^{-1} D A\right)$ and $\left.\left(C, A, B, B^{-1} D B\right)\right)$ shows that $\operatorname{tr} B C<-2$ and $\operatorname{tr} C A<-2$.

The next lemma gives an equation which corresponds, for the case treated above, to (1.2) for the case of torus.

Lemma 2.1. Let $x=-\frac{1}{2} \operatorname{tr} B C, y=-\frac{1}{2} \operatorname{tr} C A$ and $z=-\frac{1}{2} \operatorname{tr} A B$. Define the constants $a=\cos \theta_{1} \cos \theta_{4}+\cos \theta_{2} \cos \theta_{3}, b=\cos \theta_{2} \cos \theta_{4}+\cos \theta_{1} \cos \theta_{3}, c=$ $\cos \theta_{3} \cos \theta_{4}+\cos \theta_{1} \cos \theta_{2}$ and

$$
d=4 \cos \theta_{1} \cos \theta_{2} \cos \theta_{3} \cos \theta_{4}+\cos ^{2} \theta_{1}+\cos ^{2} \theta_{2}+\cos ^{2} \theta_{3}+\cos ^{2} \theta_{4}-1 .
$$


Then the Teichmüller space for the signature $\left(0 ; 2 \theta_{1}, 2 \theta_{2}, 2 \theta_{3}, 2 \theta_{4}\right)$ is represented by the sublocus $T$ satisfying $x>1, y>1$ and $z>1$ of the equation

$$
x^{2}+y^{2}+z^{2}-2 x y z+2 a x+2 b y+2 c z+d=0 .
$$

The equation (2.5) is obtained by a lengthy calculation starting from the equation $\operatorname{tr}\left[(A B C)^{2}\right]=\operatorname{tr} D^{2}$ and expressing the left-hand side as a function of $x, y$ and $z$.

In order to see that $T$ represents the Teichmüller space, consider the mapping $\varphi(l, s)=(x, y, z)$ which sends the Fenchel-Nielsen coordinates $(l, s)$ to $T$. From the formulas $z=\cosh l / 2, x=\alpha \cosh s+\beta, y=\gamma \cosh (s-l / 2)+\delta$ with $\alpha, \beta, \gamma$ and $\delta$ independent of $s$, it follows that $\varphi$ is injective and a real-analytic diffeomorphism.

We calculate the area form (1.1), where $l$ and $s$ are the Fenchel-Nielsen coordinates, in terms of $x, y$ and $z$. Since $z=-\frac{1}{2} \operatorname{tr} A B=\cosh \frac{l}{2}$ and $x=-\frac{1}{2} \operatorname{tr} B C$, we get $l=2 \log \left(z+\sqrt{z^{2}-1}\right), s=\log \left(L+\sqrt{L^{2}-1}\right)$ with

$$
L=\frac{x+\cos \theta_{1} \cos \theta_{4}-u \xi v^{-1} \eta^{-1} \sin \theta_{1} \sin \theta_{4}}{v^{-1} \eta^{-1} \sin \theta_{1} \sin \theta_{4}} .
$$

Since $\partial(l, s) / \partial(z, x)=(\partial l / \partial z) \cdot(\partial s / \partial x)$,

$$
\omega_{W P}=\frac{4 d z \wedge d x}{\sqrt{x^{2} z^{2}-x^{2}-z^{2}-2 b x z-2 a x-2 c z+b^{2}-d}} .
$$

In order to make our description simple, we will often call a boundary curve of length $\theta$ a cone point of angle $i \theta$.

By (2.5) the denominator of (2.6) equals $x z-y-b$ and again by (2.5), $d z=$ $z_{x} d x+z_{y} d y, d x=x_{y} d y+x_{z} d z$, where

$$
z_{y}=\frac{x z-y-b}{z-x y+c}, \quad x_{y}=\frac{x z-y-b}{x-y z+a} .
$$

Therefore

$$
\omega_{W P}=\frac{4 d z \wedge d x}{x z-y-b}=\frac{4 d x \wedge d y}{x y-z-c}=\frac{4 d y \wedge d z}{y z-x-a} .
$$

Originally (2.6) is obtained from the Fenchel-Nielsen coordinates associated to the simple closed curve which separates the cone points of angles $\theta_{1}$ and $\theta_{2}$ from those of angles $\theta_{3}$ and $\theta_{4}$; see Figures $1 \mathrm{a}$ and $1 \mathrm{~b}$. But the symmetry in $(x, a),(y, b)$ and $(z, c)$ manifested in the expression (2.7) implies that (2.6) can be obtained also from the simple closed curve separating the cone points of angles $\theta_{1}$ and $\theta_{3}$ from those of angles $\theta_{2}$ and $\theta_{4}$ and the one separating the cone points of angles $\theta_{1}$ and $\theta_{4}$ from those of angles $\theta_{2}$ and $\theta_{3}$. Since $\mathbf{T}(0 ; \pi, \pi, \pi, \theta)=\mathbf{T}(1 ; 2 \theta)$ (see Section 5 ), we see also that the 2 -form $d l \wedge d s$ on $\mathbf{T}(1 ; 2 \theta)$ does not depend on a particular choice of Fenchel-Nielsen coordinates $(l, s)$. This fact can be used to show that a 2 -form on any Teichmüller space defined analogously to Wolpert's formula of Weil-Petersson form [12] is invariant under the action of the mapping class group as long as the Teichmüller space admits global Fenchel-Nielsen coordinates.

Let $S$ be an oriented closed surface of genus $g$ and $P=\left\{x_{1}, \ldots, x_{n}\right\}$ a fixed set of $n$ points on $S$. Let $\left(g ; \theta_{1}, \ldots, \theta_{n}\right)$ be the signature of a hyperbolic surface. A point of the Teichmüller space $\mathbf{T}\left(g ; \theta_{1}, \ldots, \theta_{n}\right)$ is a class of marked hyperbolic surfaces $(R,[f])$ where $[f]$ is the isotopy class of an orientation-preserving homeomorphism $f: S \backslash P \rightarrow R \backslash\{$ cone points $\}$ such that $f$ sends the neighborhood of $x_{i}$ to the singularity of type $\theta_{i}$. 
We assume that $\left(g ; \theta_{1}, \ldots, \theta_{n}\right)$ is a signature such that any hyperbolic surface $R$ with this signature satisfies the following property: Any simple closed curve $c$ on $R \backslash\{$ cone points can be deformed to a unique geodesic curve under a homotopy which meets no cone points. There is an exceptional case where $c$ bounds a disk with two cone points of angle $\pi$. In this case $c$ is deformed to the degeneration of a simple closed curve and goes between the cone points along a geodesic arc back and forth. Then, given a curve-system $\left(c_{1}, \ldots, c_{d}\right), d=3 g-3+n$, which decomposes $S$ into pairs of pants, we can define the Fenchel-Nielsen coordinates $\left(l_{1}, \ldots, l_{d}, s_{1}, \ldots, s_{d}\right)$ on the Teichmüller space $\mathbf{T}\left(g ; \theta_{1}, \ldots, \theta_{n}\right)$ and also a 2 -form by

$$
\omega_{W P}=\sum_{i=1}^{d} d l_{i} \wedge d s_{i}
$$

Our aim is to show that the form (2.8) is independent of the choice of the curvesystem. Let $\mathcal{S}=\left(c_{1}, \ldots, c_{d}\right)$ and $\mathcal{S}^{\prime}=\left(c_{1}^{\prime}, \ldots, c_{d}^{\prime}\right)$ be two curve-systems as above such that $c_{j}=c_{j}^{\prime}$ for all indices $j$ except for one $i$. Namely, $\mathcal{S}^{\prime}$ is a result of a Thurston-Hatcher type simple move on $\mathcal{S}([4)$. They define Fenchel-Nielsen coordinates $\left(l_{1}, \ldots, l_{d}, s_{1}, \ldots, s_{d}\right)$ and $\left(l_{1}^{\prime}, \ldots, l_{d}^{\prime}, s_{1}^{\prime}, \ldots, s_{d}^{\prime}\right)$ respectively, such that $l_{j}=l_{j}^{\prime}$ and $s_{j}=s_{j}^{\prime}$ for all $j \neq i$. Fix $\left\{\left(l_{j}^{0}, s_{j}^{0}\right)\right\}_{j=1, j \neq i}^{d} \in\left(\mathbf{R}_{+} \times \mathbf{R}\right)^{d-1}$. Then the subspace of $\mathbf{T}\left(g ; \theta_{1}, \ldots, \theta_{d}\right)$ determined by $l_{j}=l_{j}^{0}, s_{j}=s_{j}^{0}, j=1, \ldots, i-1, i+1, \ldots, d$, can be identified with a two-dimensional Teichmüller space. For example, if $c_{i_{1}}=$ $c_{i_{1}}^{\prime}, \ldots, c_{i_{4}}=c_{i_{4}}^{\prime}$ separate $c_{i}$ (resp. $c_{i}^{\prime}$ ) from the rest in $\mathcal{S}$ (resp. $\mathcal{S}^{\prime}$ ), then the latter Teichmüller space is $\mathbf{T}\left(0 ; \hat{\theta}_{1}, \hat{\theta}_{2}, \hat{\theta}_{3}, \hat{\theta}_{4}\right)$ with $\hat{\theta}_{k}=i 2^{-1} l_{i_{k}}$. Both $\left(l_{i}, s_{i}\right)$ and $\left(l_{i}^{\prime}, s_{i}^{\prime}\right)$ give Fenchel-Nielsen coordinates for this two-dimensional Teichmüller space and so $d l_{i} \wedge d s_{i}=d l_{i}^{\prime} \wedge d s_{i}^{\prime}$, as we have seen above. Since $\left\{\left(l_{j}^{0}, s_{j}^{0}\right)\right\}_{j=1, j \neq i}^{d}$ are arbitrary, we obtain

$$
\sum_{i=1}^{d} d l_{i} \wedge d s_{i}=\sum_{i=1}^{d} d l_{i}^{\prime} \wedge d s_{i}^{\prime} .
$$

For any pair of curve-systems $\mathcal{S}$ and $\mathcal{S}^{\prime}$, there exists a finite chain of curve-systems $\mathcal{S}=\mathcal{S}_{1}, \mathcal{S}_{2}, \ldots, \mathcal{S}_{q}=\mathcal{S}^{\prime}$ such that $\mathcal{S}_{j+1}$ is obtained from $\mathcal{S}_{j}$ by a simple move for $j=1, \ldots, q-1$; see [4], [6]. We conclude that the form (2.8) is independent of the choice of curve-system which decomposes $S$ into pairs of pants.

Theorem 2.2. The 2-form $\omega_{W P}$ is invariant under the action of the mapping class group.

This theorem is known for the case of hyperbolic surfaces without cone points and almost trivial because (2.8) is Wolpert's formula for the Weil-Petersson Kähler form [12]. Our statement of the theorem includes the case of conical hyperbolic surfaces with boundary and the following proof is elementary.

Proof of Theorem 2.2. Since the action of each mapping class is a composite of full (or Dehn) twists and half-twists about a finite number of simple closed curves, it suffices to show that $\omega_{W P}$ is invariant under the twist about a simple closed curve. Here the half-twist is performed only about the boundary curve of a disk which contains two cone points of the same angle and avoids all other cone points.

Let $c$ be a simple closed curve on $S \backslash P$. Then there is a curve-system $\mathcal{S}=$ $\left(c_{1}, \ldots, c_{d}\right)$ with $c=c_{1}$ which decomposes $S$ into pairs of pants. Let $\left(l_{1}, \ldots, l_{d}\right.$, 


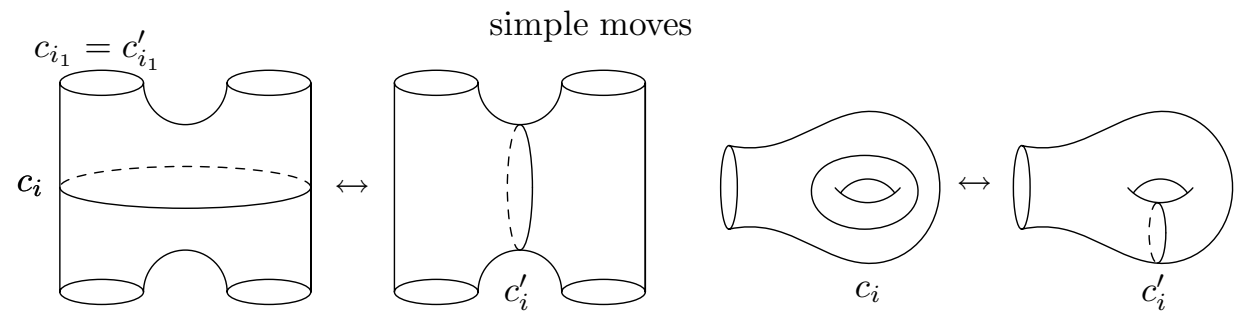

FIGURE 2.

$s_{1}, \ldots, l_{d}$ ) be the Fenchel-Nielsen coordinates defined by $\mathcal{S}$. Express $\omega_{W P}$ as in (2.8). Then invariance of $\omega_{W P}$ is obvious, because the full twist about $c$ causes the translation $s_{1} \mapsto s_{1} \pm l_{1}$ and the half-twist causes $s_{1} \mapsto s_{1} \pm \frac{1}{2} l_{1}$.

\section{ACtion OF THE MAPPING ClASS GROUP}

If, for instance, $y$ and $z$ are fixed, then (2.5) regarded as a quadratic equation in $x$ has the pair of roots $x$ and $2 y z-x-2 a$. Thus the transformations in $\mathbf{R}_{+}^{3}$ defined by

$$
\begin{aligned}
& \alpha:(x, y, z) \longmapsto(2 y z-x-2 a, y, z), \\
& \beta:(x, y, z) \longmapsto(x, 2 z x-y-2 b, z), \\
& \gamma:(x, y, z) \longmapsto(x, y, 2 x y-z-2 c)
\end{aligned}
$$

exchange the pairs of roots and preserve the surface $T$. The geometric meaning of these transformations is as follows. Let $p_{A}, p_{B}, p_{C}$ and $p_{D}$ denote the cone points of angles $\theta_{1}, \theta_{2}, \theta_{3}$ and $\theta_{4}$, respectively. Then $\alpha$ corresponds to the topological reflection of the sphere in a Jordan curve going through $p_{A}, p_{B}, p_{D}, p_{C}$ in this order; see Figure 3. Likewise $\beta$ corresponds to the topological reflection in a Jordan curve going through $p_{B}, p_{C}, p_{D}, p_{A}$ in this order and $\gamma$ to the topological reflection in a Jordan curve going through $p_{C}, p_{A}, p_{D}, p_{B}$ in this order. Then $\beta \cdot \alpha$ is a Dehn twist. In the generic case, the group generated by $\alpha, \beta$ and $\gamma$ has the same action as the mapping class group $\mathcal{M C}$. If two of $\theta_{1}, \theta_{2}, \theta_{3}$ and $\theta_{4}$ are equal, $\mathcal{M C}$ contains classes of mappings which exchange cone points of the same angle. Thus the group has the same action as a subgroup of $\mathcal{M} C$ of finite index. For the rest of this paper, we consider the generic case. We shall remark on the special case at the end of Section 4.

Let $V: T \rightarrow \mathbf{R}$ be $V(x, y, z)=x+y+z$ and let $\Delta^{\prime} \subset T$ be the set where conditions $V(x, y, z)<V(\alpha(x, y, z)), V(x, y, z)<V(\beta(x, y, z))$ and $V(x, y, z)<$ $V(\gamma(x, y, z))$ are satisfied. These conditions are equivalent to $x<y z-a, y<z x-b$ and $z<x y-c$. We show that $\Delta^{\prime}$ is a fundamental domain for $\mathcal{M} C$. Since the action of $\mathcal{M} C$ is discontinuous and since $V(x, y, z)$ tends to $+\infty$ as $(x, y, z) \in T$ tends to the boundary of $T$, the function $V$ achieves minima in each orbit $\mathcal{M} C(p)$, $p \in T$ and if $q \in \mathcal{M} C(p)$ represents a minimum, then $q$ must be in the closure of $\Delta^{\prime}$. Hence each $\mathcal{M C}$-orbit meets the closure of $\Delta^{\prime}$. Next we show that two distinct points of $\Delta^{\prime}$ are not $\mathcal{M C}$-equivalent. First observe that the three subsets $\Delta_{x}^{\prime}=\{(x, y, z) \in T: x \geq y z-a\}, \Delta_{y}^{\prime}=\{(x, y, z) \in T: y \geq z x-b\}$ and $\Delta_{z}^{\prime}=\{(x, y, z) \in T: z \geq x y-b\}$ are mutually disjoint. Now assume that $p \in \Delta^{\prime}$ and that $\delta(p) \in \Delta^{\prime}$ for some non-trivial $\delta \in \mathcal{M} C$. Express $\delta$ by a shortest word in 

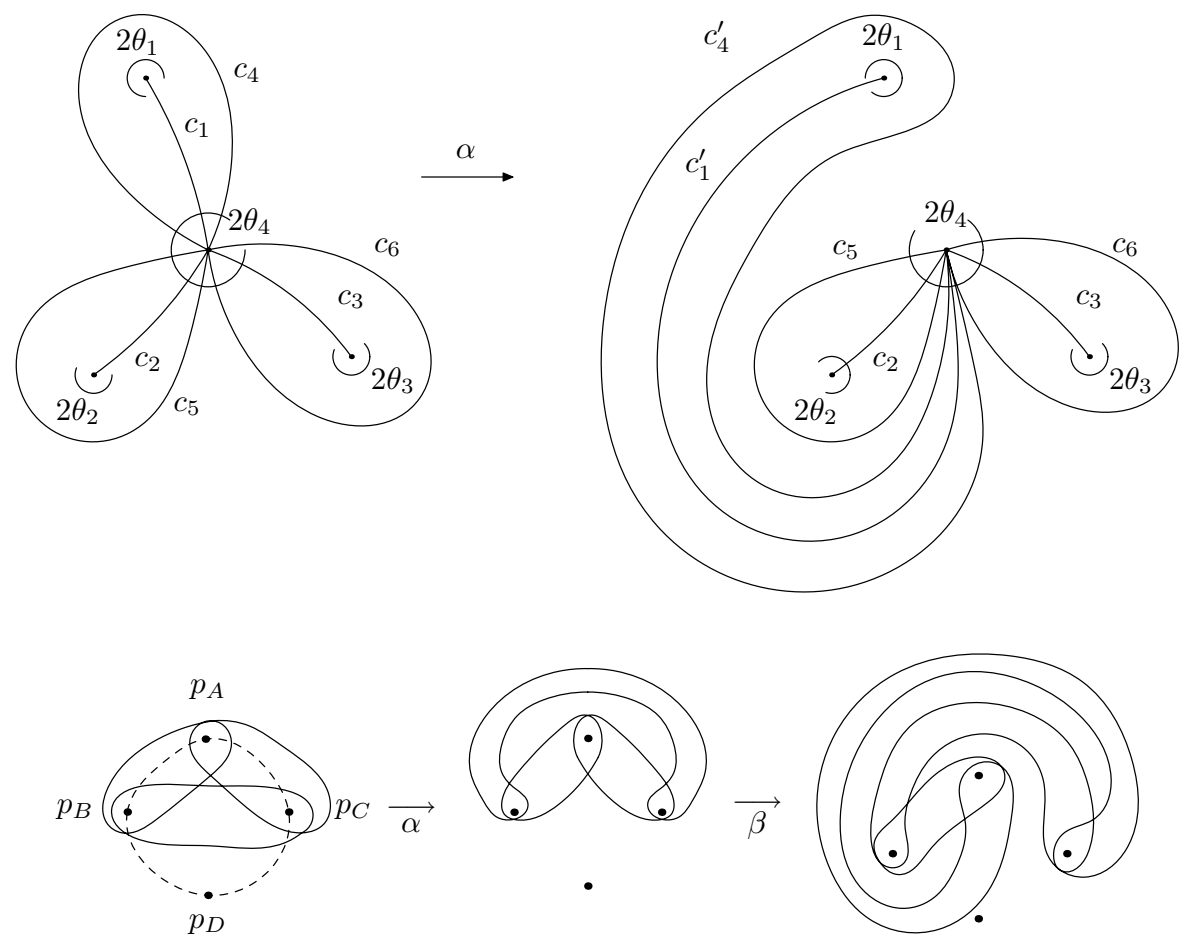

FiguRE 3.

$\{\alpha, \beta, \gamma\}: \delta=\delta_{n} \delta_{n-1} \cdots \delta_{1}, \delta_{i} \in\{\alpha, \beta, \gamma\}$. Set $p_{0}=p$ and inductively $p_{i}=\delta_{i}\left(p_{i-1}\right)$, $(i=1, \ldots, n)$. Since $V(p)<V\left(p_{1}\right)$ and $V\left(p_{n-1}\right)>V(\delta(p))$, there exists $i$ such that $V\left(p_{i-1}\right) \leq V\left(p_{i}\right)$ and $V\left(p_{i}\right)>V\left(p_{i+1}\right)$. Suppose that $\delta_{i}=\alpha$. Then $\delta_{i+1} \neq \alpha$ due to the shortest word-representation of $\delta$ because $\alpha^{2}=1$. Let, for example, $\delta_{i+1}=\beta$. Then $V\left(p_{i-1}\right) \leq V\left(p_{i}\right)$ means $p_{i} \in \Delta_{x}^{\prime}$ and $V\left(p_{i}\right)>V\left(p_{i+1}\right)$ means $p_{i} \in \Delta_{y}^{\prime}$, which is a contradiction. Other cases can be treated similarly. In conclusion, each $\mathcal{M C}$-orbit meets $\Delta^{\prime}$ at most once and thus $\Delta^{\prime}$ is a fundamental domain for $\mathcal{M} C$.

\section{The AREA of the moduli space For the Signature $\left(0 ; 2 \theta_{1}, 2 \theta_{2}, 2 \theta_{3}, 2 \theta_{4}\right)$}

It follows from (2.6) and symmetry in $x, y$ and $z$ shown in (2.7) and also from the discussion and results of the preceding sections, that the area of the moduli space $\mathbf{T}\left(0 ; 2 \theta_{1}, 2 \theta_{2}, 2 \theta_{3}, 2 \theta_{4}\right) / \mathcal{M C}$ is

$$
V=V\left(\theta_{1}, \theta_{2}, \theta_{3}, \theta_{4}\right)=\iint_{\Delta} \frac{4 d x d y}{\sqrt{x^{2} y^{2}-x^{2}-y^{2}-2 c x y-2 a x-2 b y+c^{2}-d}},
$$

where $\Delta$ denotes the image of $\Delta^{\prime}$ under the projection $(x, y, z) \mapsto(x, y)$, which is one-to-one in $\Delta^{\prime}$ because (2.5) and the inequality $z<x y-c$ yield

$$
z=x y-c-\sqrt{x^{2} y^{2}-x^{2}-y^{2}-2 c x y-2 a x-2 b y+c^{2}-d} .
$$

The domain $\Delta$ is bounded by three curves $C_{z}: z=x y-c, C_{y}: y=z x-b$ and $C_{x}: x=y z-a$ with $z$ satisfying (4.1); see Figure 4. 


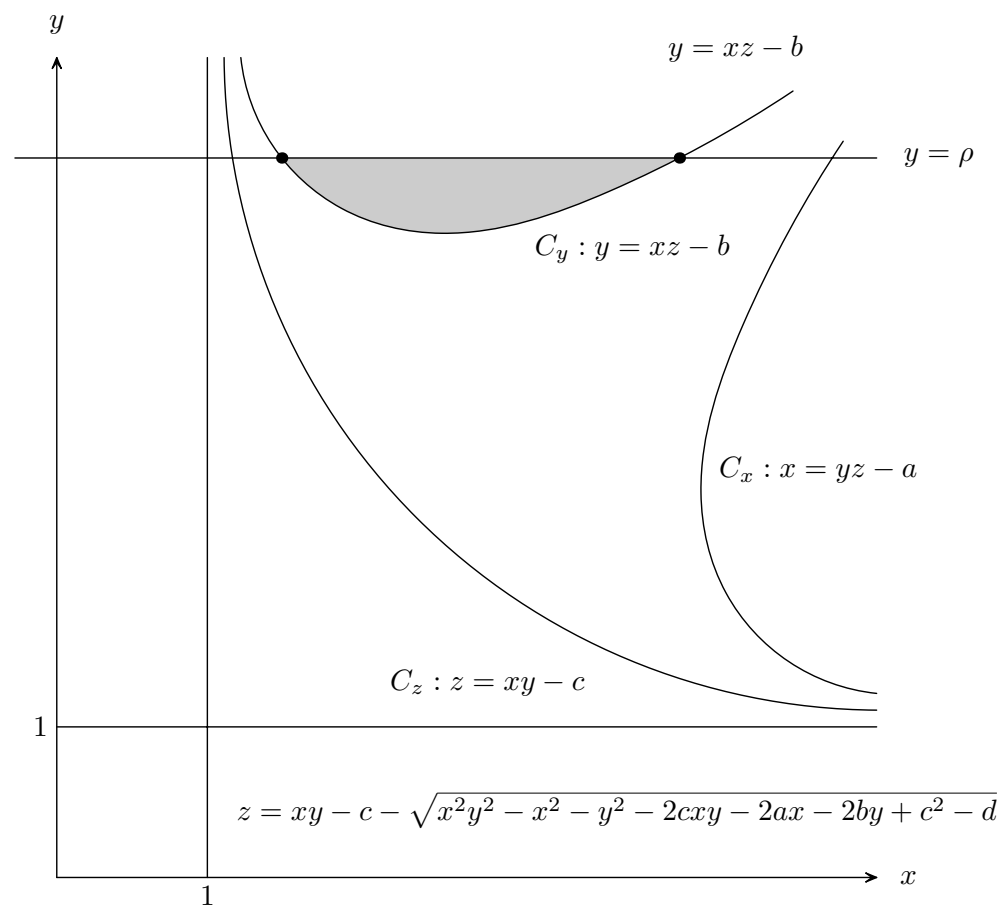

FIGURE 4.

By making a change of variables $F_{1}:(x, y) \mapsto(X, Y)$ with

$$
x=\frac{c y+a}{y^{2}-1}+X \frac{\sqrt{(c y+a)^{2}+\left(y^{2}-1\right)\left(y^{2}+2 b y-c^{2}+d\right)}}{y^{2}-1}, \quad y=Y,
$$

we obtain

$$
V=\iint_{F_{1} \Delta} \frac{4 d X d Y}{\sqrt{\left(X^{2}-1\right)\left(Y^{2}-1\right)}}
$$

The domain $F_{1} \Delta$ is bounded by $F_{1} C_{z}: X=1, F_{1} C_{x}: X=Y$ and $F_{1} C_{y}$. Unfortunately, it is not clear how to express $F_{1} C_{y}$ by a graph of an explicit function of $X$, hence we take a roundabout course to our goal. Observe, that if $(x, y) \in C_{y}$, then $y$ diverges to $+\infty$ as $x$ tends to 1 or to $+\infty$ and, if $\rho$ is chosen to be sufficiently large, then the line $y=\rho$ meets $C_{y}$ in only two points. Let $D(\rho)$ denote the domain between $y=\rho$ and $C_{y}$. Then

$V=\lim _{\rho \rightarrow+\infty}\left\{\int_{1}^{\rho}\left[\int_{X}^{\rho} \frac{4 d Y}{\sqrt{\left(X^{2}-1\right)\left(Y^{2}-1\right)}}\right] d X-\iint_{F_{1} D(\rho)} \frac{4 d X d Y}{\sqrt{\left(X^{2}-1\right)\left(Y^{2}-1\right)}}\right\}$.

We fix $\theta_{1}, \theta_{2}$ and $\theta_{3}$ and think of $V$ as a function of $\theta_{4}$. Let $V_{\rho}\left(\theta_{4}\right)$ denote the term inside the brackets of the expression above. Then only the last integral depends on $\theta_{4}$ and changing the variables back gives

$$
\frac{d V_{\rho}\left(\theta_{4}\right)}{d \theta_{4}}=-\frac{d}{d \theta_{4}} \iint_{D(\rho)} \frac{4 d x d y}{\sqrt{x^{2} y^{2}-x^{2}-y^{2}-2 c x y-2 a x-2 b y+c^{2}-d}} .
$$


The change of variables $F_{2}:(x, y) \mapsto(X, Y)$ with

$$
x=X, \quad y=\frac{c x+b}{x^{2}-1}+Y \frac{\sqrt{(c x+b)^{2}+\left(x^{2}-1\right)\left(x^{2}+2 a x-c^{2}+d\right)}}{x^{2}-1},
$$

deforms the curve $C_{y}$ to $F_{2} C_{y}: X=Y$ and $y=\rho$ to $Y=Y_{\rho}(X)$, where

$$
Y_{\rho}(X)=Y_{\rho}\left(X, \theta_{4}\right)=\frac{\left(X^{2}-1\right) \rho-(c X+b)}{\sqrt{(c X+b)^{2}+\left(X^{2}-1\right)\left(X^{2}+2 a X-c^{2}+d\right)}},
$$

and the last integral becomes

$$
\iint_{F_{2} D(\rho)} \frac{4 d X d Y}{\sqrt{\left(X^{2}-1\right)\left(Y^{2}-1\right)}}=\int_{\alpha(\rho)}^{\beta(\rho)} \frac{4}{\sqrt{X^{2}-1}} \log \frac{Y_{\rho}(X)+\sqrt{Y_{\rho}(X)^{2}-1}}{X+\sqrt{X^{2}-1}} d X,
$$

where $\alpha(\rho)=\alpha\left(\rho, \theta_{4}\right), \beta(\rho)=\alpha\left(\rho, \theta_{4}\right)$ denote the $X$-coordinates of the intersection points of $Y=X$ and $Y=Y_{\rho}(X)$. Since the integrand of the last integral vanishes at $X=\alpha(\rho)$ and $X=\beta(\rho)$, by denoting the variable $X$ by $x$ we get

$$
\begin{aligned}
&-\frac{d}{d \theta_{4}} \int_{\alpha(\rho)}^{\beta(\rho)} \frac{4}{\sqrt{x^{2}-1}} \log \frac{Y_{\rho}(x)+\sqrt{Y_{\rho}(x)^{2}-1}}{x+\sqrt{x^{2}-1}} d x \\
&=-\int_{\alpha(\rho)}^{\beta(\rho)}\left(\frac{4 \frac{\partial Y_{\rho}}{\partial \theta_{4}}\left(x, \theta_{4}\right)}{\sqrt{Y_{\rho}(x)^{2}-1}}\right) \frac{d x}{\sqrt{x^{2}-1}} .
\end{aligned}
$$

When $\rho \rightarrow+\infty$, the last integral converges locally uniformly in $\theta_{4}$ to

$$
\int_{1}^{+\infty} \frac{4}{\sqrt{x^{2}-1}}\left[\frac{\partial}{\partial \theta_{4}} \log \left((c x+b)^{2}+\left(x^{2}-1\right)\left(x^{2}+2 a x-c^{2}+d\right)\right)\right] d x .
$$

From (2.5) we obtain the factorization

$$
\begin{aligned}
& (c x+b)^{2}+\left(x^{2}-1\right)\left(x^{2}+2 a x-c^{2}+d\right) \\
& \quad=\left(x+\cos \left(\theta_{1}+\theta_{4}\right)\right)\left(x+\cos \left(\theta_{1}-\theta_{4}\right)\right)\left(x+\cos \left(\theta_{2}+\theta_{3}\right)\right)\left(x+\cos \left(\theta_{2}-\theta_{3}\right)\right) .
\end{aligned}
$$

Finally, we obtain

$$
\lim _{\rho \rightarrow \infty} \frac{d V_{\rho}}{d \theta_{4}}\left(\theta_{4}\right)=-8 \int_{1}^{+\infty} \frac{1}{\sqrt{x^{2}-1}}\left(\frac{\left(\cos \theta_{1} x+\cos \theta_{4}\right) \sin \theta_{4}}{\left(x+\cos \left(\theta_{1}+\theta_{4}\right)\right)\left(x+\cos \left(\theta_{1}-\theta_{4}\right)\right)}\right) d x .
$$

By [7, $§ 55$ (ii) $1^{\circ}$ the integral has value $-4 \theta_{4}$ and the locally uniform convergence in $\theta_{4}$ yields also $\partial V / \partial \theta_{4}=-4 \theta_{4}$. By symmetry, $V=C-2\left(\theta_{1}^{2}+\theta_{2}^{2}+\theta_{3}^{2}+\theta_{4}^{2}\right)$, and the constant $C$ can be determined to be $2 \pi^{2}$ because the area tends to 0 as the signature $(0 ; \pi / 2, \pi / 2, \pi / 2, \theta), \theta<\pi / 2$, tends to the degenerate case $(0 ; \pi / 2, \pi / 2, \pi / 2, \pi / 2)$. This gives the result claimed in Theorem 1.1 .

Remark. If two of $\theta_{1}, \theta_{2}, \theta_{3}$ and $\theta_{4}$ in a signature $\left(0 ; 2 \theta_{1}, 2 \theta_{2}, 2 \theta_{3}, 2 \theta_{4}\right)$ are equal, $\mathcal{M C}$ has a finite extension $\widetilde{\mathcal{M C}}$, which contains classes of mappings which permute cone points of the same angle. We call $\widetilde{\mathcal{M C}}$ the full mapping class group and denote by $\widetilde{\mathcal{M C}}^{+}$the subgroup of index 2 consisting of all classes of orientation preserving mappings. The areas of the spaces $\mathbf{T}\left(0 ; 2 \theta_{1}, 2 \theta_{2}, 2 \theta_{3}, 2 \theta_{4}\right) / \widetilde{\mathcal{M} C}$ and $\mathbf{T}\left(0 ; 2 \theta_{1}, 2 \theta_{2}, 2 \theta_{3}, 2 \theta_{4}\right) / \widetilde{\mathcal{M C}}^{+}$are calculated by dividing the value in (1.3) by the index $[\widetilde{\mathcal{M C}}: \mathcal{M C}]$ or by $\left[\widetilde{\mathcal{M C}}^{+}: \mathcal{M C}\right]=\frac{1}{2}[\widetilde{\mathcal{M C}}: \mathcal{M C}]$, respectively.

Case 1. If $\theta_{1}=\theta_{2}$, then $[\widetilde{\mathcal{M C}}: \mathcal{M C}]=2$. In this case $\widetilde{\mathcal{M C}}$ is obtained by adding to $\mathcal{M} C$ the class $\delta:(x, y, z) \mapsto(y, x, z)$. 
Case 2. If $\theta_{1}=\theta_{2}=\theta_{3}, \widetilde{\mathcal{M C}}$ is obtained by adding to $\mathcal{M C}$ the classes $\delta_{1}$ : $(x, y, z) \mapsto(y, x, z)$ and $\delta_{2}:(x, y, z) \mapsto(y, z, x)$. Thus $\left\langle\delta_{1}, \delta_{2}\right\rangle$ is isomorphic to the symmetry group $S_{2}$ and $[\widetilde{\mathcal{M C}}: \mathcal{M C}]=6$.

The mapping class group $\mathcal{M}(0,4)$ (by the notation in 1]) of the 4 punctured sphere $S^{2} \backslash\left\{p_{1}, p_{2}, p_{3}, p_{4}\right\}$, which is treated in detail in [1, Section 5.3] is one of the mapping class groups which has a special action on Teichmüller spaces; see [14. E. 3.17, p. 203]. For instance, choose a simple closed curve $c$ which separates the pair $p_{1}, p_{2}$ from $p_{3}$ and $p_{4}$. Then a half-twist about $c$ interchanging $p_{1}$ and $p_{2}$ can cause the same action as a half-twist about $c$ interchanging $p_{3}$ and $p_{4}$. So Case 1 includes the case where $\theta_{1}=\theta_{2}$ and $\theta_{3}=\theta_{4}$ and Case 2 includes the case where $\theta_{1}=\theta_{2}=\theta_{3}=\theta_{4}$.

\section{The ARea of the moduli SPACE of the torus With ONE SINGUlarity}

In $[8$, the area of the moduli space for the signature $(1 ; 2 \theta)$ was calculated to be

$$
\frac{1}{6}\left(\pi^{2}-\theta^{2}\right)
$$

Here we consider the mapping class group consisting of only classes of orientationpreserving mappings so that (5.1) is comparable with Wolpert's result [10.

The value in the expression (5.1) can be deduced also from the fact that $\mathbf{T}(1 ; 2 \theta)$ $=\mathbf{T}(0 ; \pi, \pi, \pi, \theta)$. This fact follows from that every hyperbolic cone surface with signature $(1,2 \theta)$ admits the hyperelliptic involution: The same matrices $A, B, C$ and $D$ as in Section 2 satisfy $A^{2}=B^{2}=C^{2}=A B C D=1$ (= the identity in $\left.P S L_{2}(\mathbf{R})\right)$ and the commutator $[A C, C B]$ equals $D^{-2}$. The map $(A, B, C, D) \mapsto$ $(A C, C B)$ induces a homeomorphism between the two Teichmüller spaces. Since $a=b=c=0$ in $(2.5)$ for $(0 ; \pi, \pi, \pi, \theta),(2.5)$ is symmetric in $x, y, z$ and therefore in (3.1), the order can be reversed to obtain orientation preserving mappings

$$
\begin{aligned}
& \alpha:(x, y, z) \longmapsto(z, y, 2 y z-x), \\
& \beta:(x, y, z) \longmapsto(x, 2 x y-z, y), \\
& \gamma:(x, y, z) \longmapsto(2 x z-y, x, z)
\end{aligned}
$$

instead of those in (3.1). Each of these transformations relates to the half-twist around a pair of points of order 2 on the sphere, which lifts to the Dehn twist along a simple loop on the torus, the two-sheeted covering surface. The transformations $\alpha$ and $\beta$ generate the mapping class group. (For more details on the action of the mapping class group, see [8]). Since $\beta \alpha(x, y, z)=(z, x, y)$ generates a cyclic subgroup of order 3 , the stabilizing subgroup of $\Delta^{\prime}$ (see [8]), the area is one-third of the area (1.3) for the signature $(0 ; \pi, \pi, \pi, \theta)$, the same value as the area of $\mathbf{T}(0 ; \pi, \pi, \pi, \theta) / \widetilde{\mathcal{M} C}+$.

\section{REFERENCES}

1. Birman, J. S., Braids, Links, and Mapping Class Groups, Ann. of Math. Studies 82, Princeton Univ. Press, Princeton, 1974. MR 51:11477 erratum MR 54:13894

2. Ford, L. R., Automorphic Functions, Second edition, Chelsea, New York, 1951.

3. Fricke R. and F. Klein, Vorlesungen über die Theorie der Automorphen Funktionen, Teubner, 1965. MR 32:1348

4. Hatcher, A. and W. Thurston, A presentation for the mapping class group of a closed orientable surface, Topology 19 (1980), 221-237. MR 81k:57008

5. Kaufmann, R, Yu. Manin and D. Zagier, Higher Weil-Petersson volumes of moduli spaces of stable n-pointed curves, Commun. Math. Phys. 181 (1996), 763-787. MR 98i:14029 
6. Luo, Feng, On Heegaard diagrams, Math. Res. Lett. 4 (1997), no. 2-3, 365-373. MR 95h:57030

7. Moriguchi, S, K. Udagawa and S. Hitotsumatsu, Mathematical formulae, I, Iwanami Shoten, Tokyo, 1989 (in Japanese).

8. Näätänen, M. and T. Nakanishi, Weil-Petersson areas of the moduli space of tori, Results in Mathematics 33 (1998), 120-133. MR 2000a:32028

9. Penner, R. C., Weil-Petersson volumes, J. Differential Geometry 35 (1992), 559-608. MR 93d:32029

10. Wolpert, S., On the Kähler form of the moduli space of once punctured tori, Comment. Math. Helv. 58 (1983), 246-256. MR 84j:32028

11. Wolpert, S., On the symplectic geometry of deformations of a hyperbolic surface, Ann. of Math. 117 (1983), 207-234. MR 85e:32028

12. Wolpert, S., On the Weil-Petersson geometry of the moduli space of curves, Amer. J. Math. 107 (1985), 969-997. MR 87b:32040

13. Zograf, P., The Weil-Petersson volume of the moduli space of punctured spheres, Mapping Class Groups and Moduli Spaces of Riemann Surfaces (C.F.Bödigheimer and R. M. Hain, eds.), Contemporary Mathematics, vol. 150, American Mathematical Society, 1993, pp. 367372. MR 94g:32030

14. Zieschang, H., Finite Groups of Mapping Classes of Surfaces, Lecture Notes in Math. 875, Springer, 1981. MR 86g:57001

Graduate School of Mathematics, Nagoya University, Furo-cho, Chikusa-ku, Nagoya 464-01, JAPAN

E-mail address: tosihiro@math.nagoya-u.ac.jp

Department of Mathematics, University of Helsinki, P.O. Box 4 (Yliopistonkatu 5), 00014 Helsinki, Finland

E-mail address: marjatta.naatanen@helsinki.fi 